\title{
Entwicklung eines ausgedehnten Basalzellkarzinoms auf Dermatofibromen
}

\author{
Development of an Extensive Basal Cell Carcinoma from Dermatofibromas
}

Autoren

Institut
B. Mülbert, O. Inhoff, L. Schmitt, N. Berkes, E. Dippel

Hautklinik/Hauttumorzentrum am Städtischen Klinikum Ludwigshafen am Rhein gGmbH
Bibliografie

DOI http://dx.doi.org/

10.1055/s-0030-1256638

Online-Publikation: 28. 7. 2011

Akt Dermatol 2011; 37:

303-305 ๑ Georg Thieme

Verlag KG Stuttgart · New York ISSN 0340-2541

\section{Korrespondenzadresse}

\section{Dr. med. Oliver Inhoff}

Hautklinik/Hauttumorzentrum

Rheinpfalz

Klinikum Ludwigshafen

Bremserstraße 79

67063 Ludwigshafen

inhoffo@klilu.de

\section{Zusammenfassung \\ $\nabla$}

Der vorliegende Fall zeigt die seltene Assoziation eines Basalzellkarzinoms mit Dermatofibromen auf eindrückliche Weise. Das Augenmerk soll hierbei auf die möglichen histopathologischen Veränderungen der Epidermis über Dermatofibromen, welche von leichter Akanthose bis zum Basalzellkarzinom reichen kann, gelenkt werden. Ein 43-jähriger Patient berichtet über seit 26 Jahren bestehende, gruppierte Hautveränderungen

\section{Einleitung}

In der Literatur liegen nur sehr wenige Fallberichte über die Assoziation von Dermatofibromen und Basalzellkarzinomen vor. Häufiger scheinen hingegen benigne Basalzellkarzinom-ähnliche epidermale Proliferationen mit Dermatofibromen assoziiert zu sein. In einer Mehrzahl dieser Fälle ist in der Literatur interessanterweise eine klonale Proliferation, wahrscheinlich sekundär durch die Inaktivierung des Tumorsuppressorgens PTCH, beschrieben worden.

\section{Patientenbeschreibung \\ $\nabla$}

\section{Anamnese}

Ein 43-jähriger Patient berichtet über seit ca. 26 Jahren bestehende, gruppiert stehende Hautläsionen an der Knieaußenseite rechts. Eine Ursache der Hautläsionen war anamnestisch nicht zu eruieren. In der Vergangenheit durchgeführte histologische Proben hatten den Befund von Histiozytomen gezeigt.

Bei der stationären Aufnahme berichtet der Patient über eine vor einigen Monaten neu aufgetretene Ulzeration im Bereich der vorbestehenden Hautläsionen. Bei einer im Juli 2010 ambulant durchgeführten Stanzexzision in diesem Bereich am rechten Knie, welche histologisch als Dermatofibrome eingeordnet werden konnten. Im Zentrum der Gruppe von Dermatofibromen kam es in den letzten Monaten zunehmend zu einer Plaquebildung und schließlich Ulzeration. Histologisch zeigte sich ein ausgedehntes sklerodermiform wachsendes Basalzellkarzinom, randlich angrenzend an umgebende Dermatofibrome. Es erfolgte eine Exzision im Gesunden mit anschließender Spalthauttransplantation und Vakuumversiegelung.

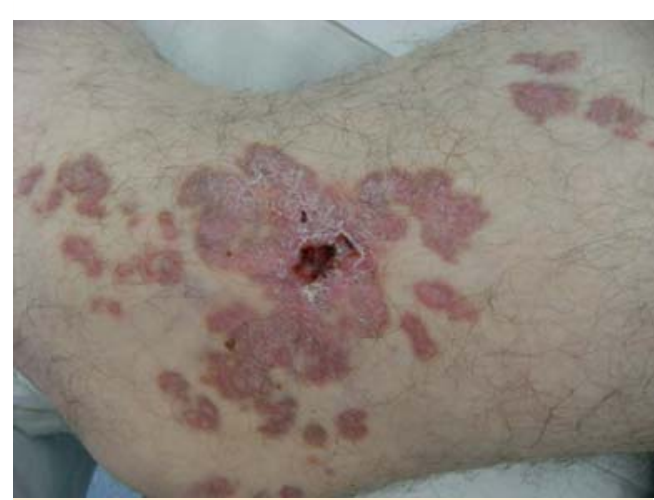

Abb. 1 Ausgangsbefund: auf einer Fläche von ca. $10 \times 20 \mathrm{~cm}$ zeigen sich gruppiert stehende, bräunlichrötliche Plaques mit leichter Schuppung, zentral zeigt sich ein ca. $2 \times 3 \mathrm{~cm}$ großer Plaque mit Ulzeration.

zeigte sich der Befund eines sklerodermiformen Basalzellkarzinoms. Es sind keine sonstigen epidermalen Malignome oder Risikofaktoren für epidermale Malignome vorbekannt.

\section{Lokalbefund}

An der Knieaußenseite rechts zeigen sich auf einer Fläche von ca. $10 \times 20 \mathrm{~cm}$ gruppiert stehende, bräunlich-rötliche Plaques mit dermaler Konsistenzvermehrung und leicht schuppiger Oberfläche. Zentral kommt es zur Konfluenz mit Ausbil- 


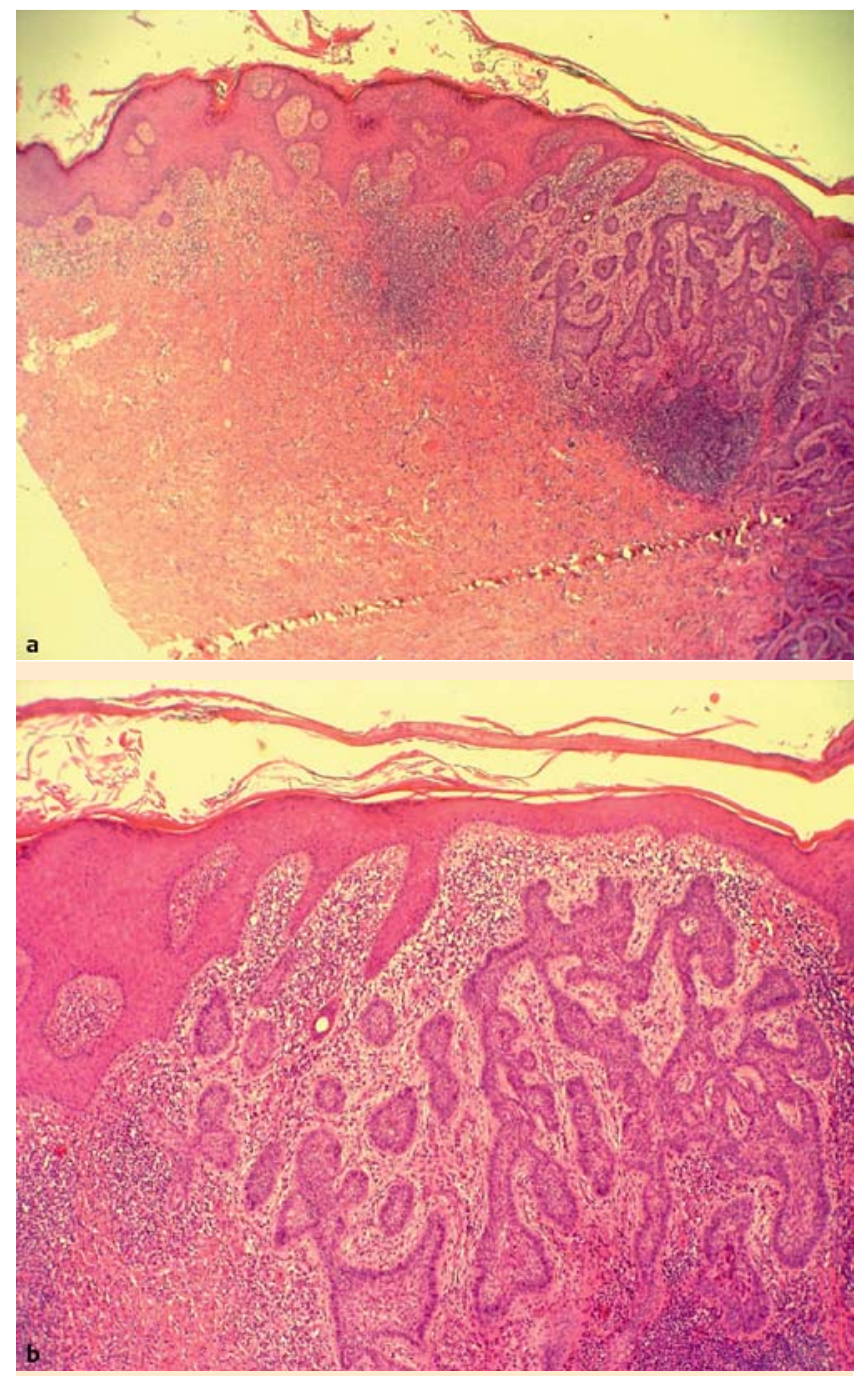

Abb. 2 a Histologisches Präparat in HE-Färbung und 20-facher VergröBerung, $\mathbf{b}$ histologisches Präparat in HE-Färbung und 40-facher VergröBerung.

dung einer ca. $3 \times 2 \mathrm{~cm}$ großen Plaque mit zentraler Ulzeration (๑ Abb. 1).

\section{Verlauf}

\section{$\nabla$}

Am Tag der stationären Aufnahme erfolgte eine histografisch kontrollierte Exzision im Bereich des ambulant bioptisch gesicherten Basalzellkarzinoms sowie drei Stanzbiopsien am rechten Knie in der Umgebung der Exzision. Makroskopisch war der

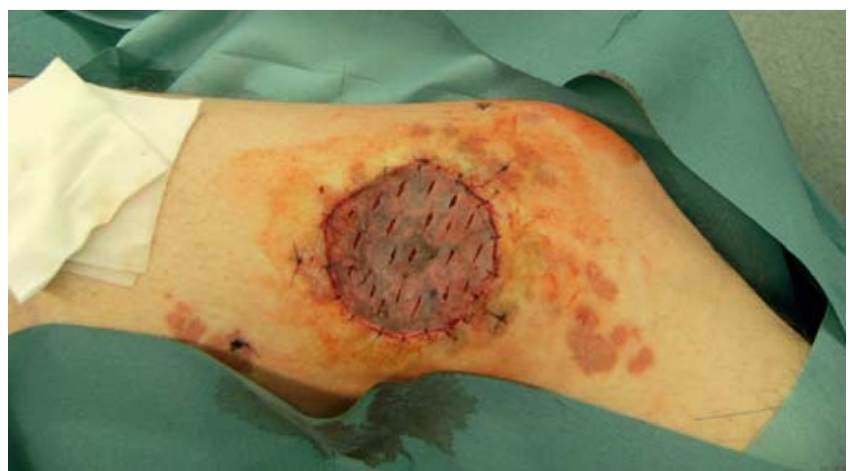

Abb. 4 Wundversorgung mittels Spalthauttransplantation vom gleichseitigen Oberschenkel.

Übergang von reaktiver Epithelproliferation und Basalzellkarzinom im Randbereich nicht erkennbar. Histologisch zeigte sich im Bereich der offenen Exzision ein Dermatofibrom mit epidermaler Proliferation und Übergang in ein Basalzellkarzinom (৫ Abb.2a,b).

Im Bereich der Stanzexisionen zeigten sich Dermatofibrome. Nach Exzision des Basalzellkarzinoms in sano erfolgte der Verschluss am rechten Bein mittels Spalthauttransplantation vom rechten Oberschenkel ( $\bullet$ Abb. $\mathbf{3}, \mathbf{4})$. Postoperativ erfolgte die Anlage eines Vakuumverbandes auf dem Transplantat mit einem Sog von $125 \mathrm{mmHg}(\boldsymbol{A} \mathbf{A b b}$. 5). Am fünften postoperativen Tag erfolgten die Entfernung des Vakuumverbandes sowie die Entlassung des Patienten mit vitalem Transplantat.

\section{Diskussion}

$\nabla$

Dermatofibrome sind gutartige dermale Neubildungen welche häufig ein Spektrum epidermaler Proliferationen ausbilden. Eine milde Akanthose findet sich am häufigsten, in einigen Fällen können sich jedoch auch follikuläre und Basalzellkarzinom-ähnliche basaloide Proliferationen ausbilden [1,2]. Die Assoziation eines Basalzellkarzinoms mit einem Dermatofibrom ist hingegen nur sehr selten dokumentiert, und über die reaktive bzw. neoplastische Natur der epidermalen Proliferationen wird kontrovers diskutiert $[3,4]$.

Bisher betrachtete man üblicherweise die epidermalen Proliferationen als Folge eines induktiven Effekts der fibrohistiozytären Zellen des Dermatofibroms auf die Epithelzellen des Haarfollikels, im Sinne eines reaktiven Geschehens [5]. Als Auslöser der reaktiven epidermalen Proliferation wurden von einigen Autoren die Kompression der Adnexstrukturen gegen die Epidermis durch das wachsende Dermatofibrom oder die Rolle löslicher
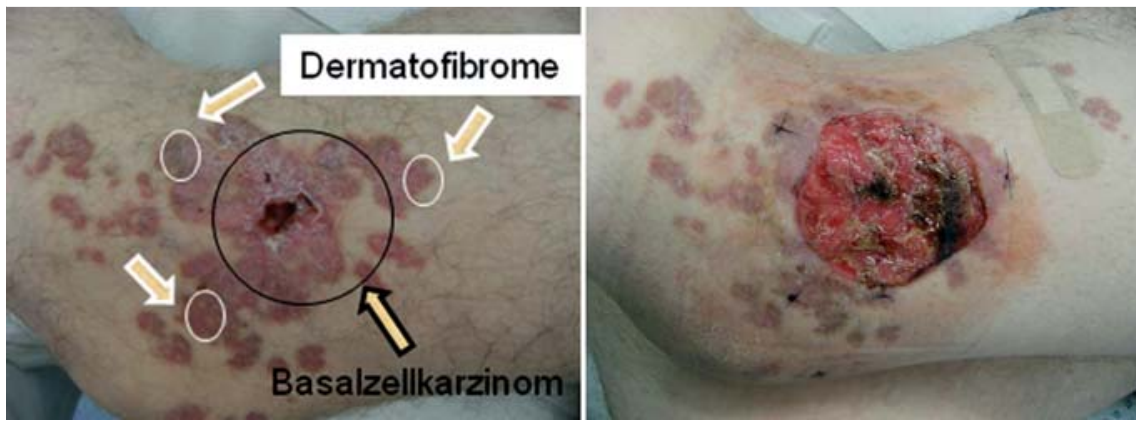

Abb. 3 Defekt nach mikrografisch kontrollierter Exzision des Basalzellkarzinoms. Makroskopisch war der Übergang von reaktiver Epithelproliferation und Basalzellkarzinom im Randbereich nicht erkennbar. 


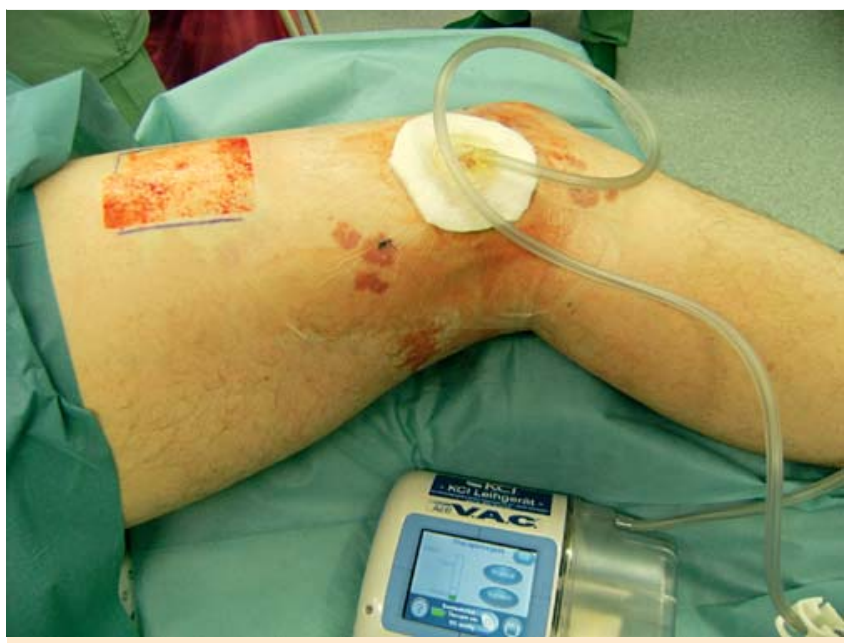

Abb.5 Anlage des Vakuumverbandes auf die Transplantatempfängerstelle.

epidermaler Wachstumsfaktoren, produziert durch dermale Zellen, postuliert [6].

Gleichwohl konnten Leong et al. den Verlust der Heterozygotie des patch (PTCH)-locus in 3 von 5 Proben mit follikulären oder Basalzellkarzinom-ähnlichen Proliferationen, aber in keiner von 5 Proben mit milder Akanthose zeigen [1]. Das PTCH-Gen hat eine Funktion als Tumorsuppressorgen in humanen Tumoren [7]. Der Verlust der Heterozygotie deutet hier auf eine klonale Proliferation in der Epidermis über Dermatofibromen hin. Der Verlust der Heterozygotie am PTCH-locus in den Basalzellkarzinom-ähnlichen Proliferationen ist vereinbar, aber nicht diagnostisch, mit einer PTCH-Inaktivierung und dem Vorhandensein eines Basalzellkarzinoms. Da PTCH-Mutationen und der Verlust der Heterozygotie am PTCH-locus auch in gutartigen Trichoepitheliomen nachgewiesen wurden, ist eine definitive Diagnosestellung durch die PTCH-Analyse jedoch nicht möglich [1].

\section{Fazit}

$\nabla$

Die Entwicklung von Basalzellkarzinomen auf Dermatofibromen ist sehr selten, aber in der Literatur wiederholt dokumentiert und sollte in der klinischen Praxis mitberücksichtigt werden. Ein Funktionsverlust des Tumorsuppressorgens patch (PTCH) scheint hierbei pathogenetisch relevant zu sein.

\section{Abstract}

\section{Development of an Extensive Basal Cell Carcinoma from Dermatofibromas \\ $\nabla$}

We present a case in which the rare association between basal cell carcinoma and dermatofibroma is demonstrated. Dermatofibromas are benign dermal tumors that frequently exhibit a spectrum of epidermal changes. Most frequent mild acanthosis is present and very rarely the diagnosis of a dermatofibroma and a basal cell carcinoma was made. A 43- year old man presented with a 26-year history of dermatofibromas on his right knee. The lesion had enlarged over time and an ulceration was observed in recent months. Surgical excision of the lesion was performed and hematoxylin-eosin staining showed a basal cell carcinoma adjoining to dermatofibroma. After micrographic surgery a split-thickness skin graft was performed.

\section{Literatur}

1 Cheng L, Amini SB, Tarif Zaim M. Follicular basal cell hyperplasia overlying dermatofibroma. Am J Surg Pathol 1997; 21: 711 - 718

2 Córdoba S, Hernández A, Romero A et al. Basal cell carcinoma overlying a dermatofibroma. Actas Dermosifiliogr 2005; 96: 612-615

3 Göppner D, Leverkus $M$. Basal cell carcinoma: from the molecular understanding of the pathogenesis to targeted therapy of progressive disease. J Skin Cancer 2011; Article ID 650258, Epub 2010 Dec 29

$4 \mathrm{Han} \mathrm{HK}$, Huh CH, Cho K. Proliferation and differentiation of keratinocytes in hyperplastic epidermis overlying dermatofibroma. Immunohistochemical characterization. Am J Dermatopathol 2001; 23: 90 - 98

5 Leong PM, Kauffman CL, Moresi JM et al. Basal cell carcinoma-like epidermal changes overlying dermatifibromas often reveal loss of heterozygosity in the PTCH gene. J Invest Dermatol 1999; 113: 279-280

6 Morgan M, Howard H, Everett MA. Epithelial induction in dermatofibroma: a role for the epidermal growth factor (EGF) receptor. Am J Dermatopathol 1997; 19: $35-40$

7 Rosmaninho A, Farrajota P, Peixoto C et al. Basal cell carcinoma overlying a dermatofibroma: a revisited controversy. Eur J Dermatol 2011; 21: $137-138$ 